\title{
Breaking Bad News in the Emergency Department: How do the Patients Want it?
}

\author{
Ramazan Guven' (D), Eylem Kuday Kaykisiz² (D) Hatice Onturk³ (D), Muhammed Ikbal Sasmaz ${ }^{4}$ (D) Asim Ari ${ }^{5}$ (D), Gokhan Eyupoglu² (D), \\ Ayse Gurol Parlak ${ }^{6}$ \\ ${ }^{1}$ Clinic of Emergency Medicine, Tarsus State Hospital, Mersin, Turkey \\ ${ }^{2}$ Clinic of Emergency Medicine, Bitlis State Hospital, Bitlis, Turkey \\ ${ }^{3}$ Department of Nursing, Bitlis Eren University School of Nursing, Bitlis, Turkey \\ ${ }^{4}$ Department of Emergency Medicine, Yuzuncu Yil University School of Medicine, Van, Turkey \\ ${ }^{5}$ Department of Emergency Medicine, Health Sciences University Dr. Ersin Arslan Training and Research Hospital, Gaziantep, Turkey \\ ${ }^{6}$ Department of Pediatric Nursing, Ataturk University School of Medicine, Erzurum, Turkey
}

Cite this article as: Guven R, Kaygisiz EK, Onturk H, Sasmaz MI, Ari A, Eyupoglu G, et al. Breaking Bad News in the Emergency Department: How do the Patients Want it? Eurasian J Emerg Med. 2018; 17 (3): 97-102.

\begin{abstract}
Aim: When breaking bad news (BBN) is not managed correctly, the negative impact on patients and patient's relatives is much greater. The aim of the present study was to investigate the thoughts of the patient/patient's relatives about how BBN should be given in four hospitals located in the eastern region of Turkey.

Materials and Methods: In this cross-sectional, multicenter study, a total of 760 patients were included using a six-item questionnaire. Participants were divided into two groups according to educational status as high school and below (Group 1) and university and above (Group 2). The difference between the groups was determined according to $p<0.05$ level of significance.

Results: There was a statistically significant difference between the two groups in terms of the answers to items about how, where, and to whom should a doctor tell the death and also possible negative situation of a patient to his/her relatives. All participants were asked where they preferred to stay in the emergency department when cardiopulmonary resuscitation (CPR) was needed in one of the family members. Of the participants, $47.4 \%(n=360)$ reported that they preferred waiting in a seat close to the room where CPR was performed.

Conclusion: The emergency physician should break the bad news considering the educational status of the patient/patient's relatives. BBN should be carried out by sitting down face-to-face with the patients or family members in a room where no other patients are present. In contrast to some literature data, patient's relatives are more likely to wait near the CPR room instead of watching CPR.
\end{abstract}

Keywords: Breaking bad news, emergency department, patients

\section{Introduction}

Bad news is defined by Robert Buckman as "any news that adversely and seriously affects an individual's view of his or her present and future" (1-3). Breaking bad news (BBN) in the emergency department
(ED) is a common and routine practice performed practically every day by physicians (4). BBN to patients or their family members is one of the most challenging aspects of medical practice (5). In our country, BBN to a patient is not in a standardized approach and also the relatives of the patients try to hide the disease from the patient. In

ORCID IDs of all authors: R.G. 0000-0003-4129-8985; E.K.K. 0000-0002-4699-4691; H.O. 0000-0002-6206-2616; M.I.S. 0000-0002-32673184; A.A. 0000-0001-7710-5191; G.E. 0000-0001-8583-3690. 
the study conducted by Atesci et al. (6), they concluded that more than half of the patients are not aware that they have cancer. This puts emergency physicians in a difficult situation in emergency management and in communication with these patients who are often admitted to the ED.

Breaking bad news is an important and difficult task for any physician. A physician should be able to communicate effectively and should take a patient-family-centered approach (4). Bad news includes a serious illness or clinical condition and the possibility of death. This news may lead to feelings of abandonment, loneliness, and loss of control on the patient or their family members (2).

Informing family members or patients about the bad news is a highly stressful experience for emergency physicians (7). In this evidence-based era, it is imperative that all healthcare be redesigned from the perspective of the patient (5). A physician is expected to be able to disclose bad news and evaluate patients' request for it (1). There is a need for effective communication in health care (8). Effective communication skills hold the key to solve such complex issues of clinical practice as a well-communicated message though tragic (5).

There is no standard answer about how this should be when the patient and their family members have to receive bad news. This can vary according to the educational status, occupation, and culture of the patient and their family members (9-11). People with different cultural backgrounds may show different attitudes toward disclosing bad news $(1,3)$. The purpose of the present study was to explore the patients' preferences toward being informed about the bad news in the ED.

\section{Materials and Methods}

This cross-sectional study was conducted on patients admitted to the ED of four hospitals in eastern Turkey from March 2017 to June 2017. A total of 760 patients $>18$ years old who were referred to the ED of four hospitals were enrolled in the study. All subjects consented to participate in the study. Exclusion criteria were refusing participation, disability to talk and communicate, presence of cognitive disorders, alcohol or drug abuse, and patients who need urgent intervention.

The questionnaire was established based on the literature review and after examining its content validity by a number of psychiatrists. Patients' view and variables affecting how to break the bad news were discussed in the questionnaire. A questionnaire that contains the study form and consists of two parts was used. The first part includes items for sociodemographic data, whereas the second part includes items for the preferences of patients about bad news (Table 1).

The study was approved by the Bitlis Eren University Ethics Committee (no. 2017/02-V) and conducted in accordance with the principles of the Declaration of Helsinki. All participants were informed about the nature of the study. Written informed consent was obtained from the participants. Then, they were interviewed, and a questionnaire was completed for each of them by the interviewers.

\section{Statistical analysis}

Statistical analysis was performed using the Statistical Package for the Social Science 22.0 software (IBM SPSS Corp.; Armonk, NY, USA). Descriptive data were expressed as median (min-max) values, whereas categorical data were expressed as number ( $\mathrm{n}$ ) and frequency (\%). The significant differences in frequency between the groups were compared using the chi-square test. For testing reliability, the Cronbach's alpha coefficient was used to identify the internal consistency of the scales. A $p$ value of $<0.05$ was considered statistically significant.

\section{Results}

Of a total of 760 patients included in the study, $42.6 \%(n=324)$ were females, and $57.4 \%(n=436)$ were males. The median age of the patients was 29 (range 23-38) years. Participants were divided into two groups according to their educational status. The first group consisted of illiterate and primary and high school graduates (67.9\%, $\mathrm{n}=516$ ), whereas the second group consisted of university graduates and doctorate degrees $(32.1 \%, \mathrm{n}=244)$.

The Cronbach's alpha value was 0.613 in the reliability analysis for the second part of the questionnaire that includes items questioning the preferences of the patient about BBN.

Table 2 shows the answers to the questionnaires and the frequency rates. In response to the question "How do you want your doctor to communicate with you?", which was the first item of the part related to BBN in the questionnaire, $56.8 \%(n=432)$ of the participants answered as "formally and gently".

In response to the second item "How should a doctor tell a possible negative situation to his/her patient?", $51.2 \%(n=389)$ of the participants answered as 'by explaining the cause of death with medical details.'

In response to the third item "How should a doctor tell the death of the patient to his/her relatives?", $42.5 \%(n=323)$ of the participants answered as "by keeping the values of faith in the foreground."

In response to the fourth item "To whom should a doctor tell the death of the patient?", $44.6 \%(n=339)$ of the participants answered as 'to one of the family members.'

In response to the fifth item "Where should a doctor tell the death of the patient?", $56.3 \%(n=428)$ of the participants answered as "in a separate room where no other patients are present by sitting down face to face with the patient's relatives."

In response to the sixth item "Where should the patient's relative(s) be while the doctor is performing resuscitation (bring back to life efforts, heart massage etc.) to the patient in the cardiopulmonary resuscitation (CPR-bring back to life efforts) room?", $47.4 \%(n=360)$ of the participants answered as "by waiting in a seat close to the room where CPR is performed".

Table 2 shows the presence of difference in terms of answers to the questionnaire of the participants who were evaluated into two (first and second) groups according to educational status and its level of 
Table 1. The form of the study entitled 'the preferences of patients about breaking bad news who admitted to the emergency department.'
a. Sincerely as my relative
b. Formally and gently
c. It does not matter

1. How do you want your doctor to communicate with you?

\section{How should a doctor tell a possible negative situation to his/her patient?}

a. Directly

b. By keeping the values of faith in the foreground

c. By explaining the condition of the disease and how this was developed

d. It does not matter

\section{How should a doctor tell the death of the patient to his/her family members?}

a. Directly. (We could not save your patient. We are sorry, our sincere condolences.)

b. By keeping the values of faith in the foreground (Hello. My name is $\mathrm{Dr}$ _ Mr/Mrs _. My specialty is _. We have done all necessary interventions for him/her. But it is destiny, so it is written in destiny, it is necessary to submit to fate. We could not save your patient. We are sorry, our sincere condolences.)

c. By explaining the cause of death with medical details (Hello. My name is $\mathrm{Dr} \ldots$, Mr/Mrs _. My specialty is _. He/she was brought to the emergency service in the condition of _. He/she had _ illnesses. In his/her tests (laboratory)/imaging results, _ was/were detected. We have done _ interventions for _ _ hour/minute. But we could not save Mr/Mrs _ . We could not save your patient. We are sorry, our sincere condolences.)

d. It does not matter

\section{To whom should a doctor tell the death of the patient?}

a. To one of the family members

b. To one of the relatives

c. To all family members to be present

d. It does not matter

\section{Where should a doctor tell the death of the patient?}

a. In front of the reanimation room, by standing

b. In any part (room) of the emergency department

c. In a separate room where no other patients are present, by sitting down face-to-face with family members

d. It does not matter

6. Where should the family members be while the doctor is performing resuscitation (bring back to life efforts, heart massage etc.) to the patient in the cardiopulmonary resuscitation (CPR-bring back to life efforts) room?

a. In the resuscitation room (by seeing the condition of the patient and the medical interventions performed)

b. By waiting in front of the CPR room

c. By waiting in a seat close to the room where CPR is performed

d. It does not matter

significance. Accordingly, there was a statistically significant difference between the two groups in terms of the answers to items about how, where, and to whom should a doctor tell the death of a case and also possible negative situation of a patient to his/her relatives ( $p<0.001, p<0.001, p<0.001$, and $p<0.001$, respectively).

\section{Discussion}

When BBN is not managed correctly, the negative impact on patients and patient's relatives is much greater. BBN is an important and difficult task for any physician. A physician should be able to communi- cate effectively and should take a patient-family-centered approach. In the last few decades, the traditional paternalistic model of patient care has been replaced by one that emphasizes patient autonomy, empowerment, and full disclosure $(12,13)$. Today, telling the truth is considered as a doctors' ethical duty and a patients' legal right, and patients play a major role in making decisions about their health and therapeutic procedures $(1,12)$. Thus, doctors have a legal duty to break the bad news to patients and their families. It is truly necessary and requires skill on the part of health care professionals. Thus, BBN is difficult, unpleasant, and uncomfortable. To improve such skills, guidance on how to systematize BBN and make it less traumat- 
Table 2. The responses of patient/patient's relatives to questionnaire items according to educational status

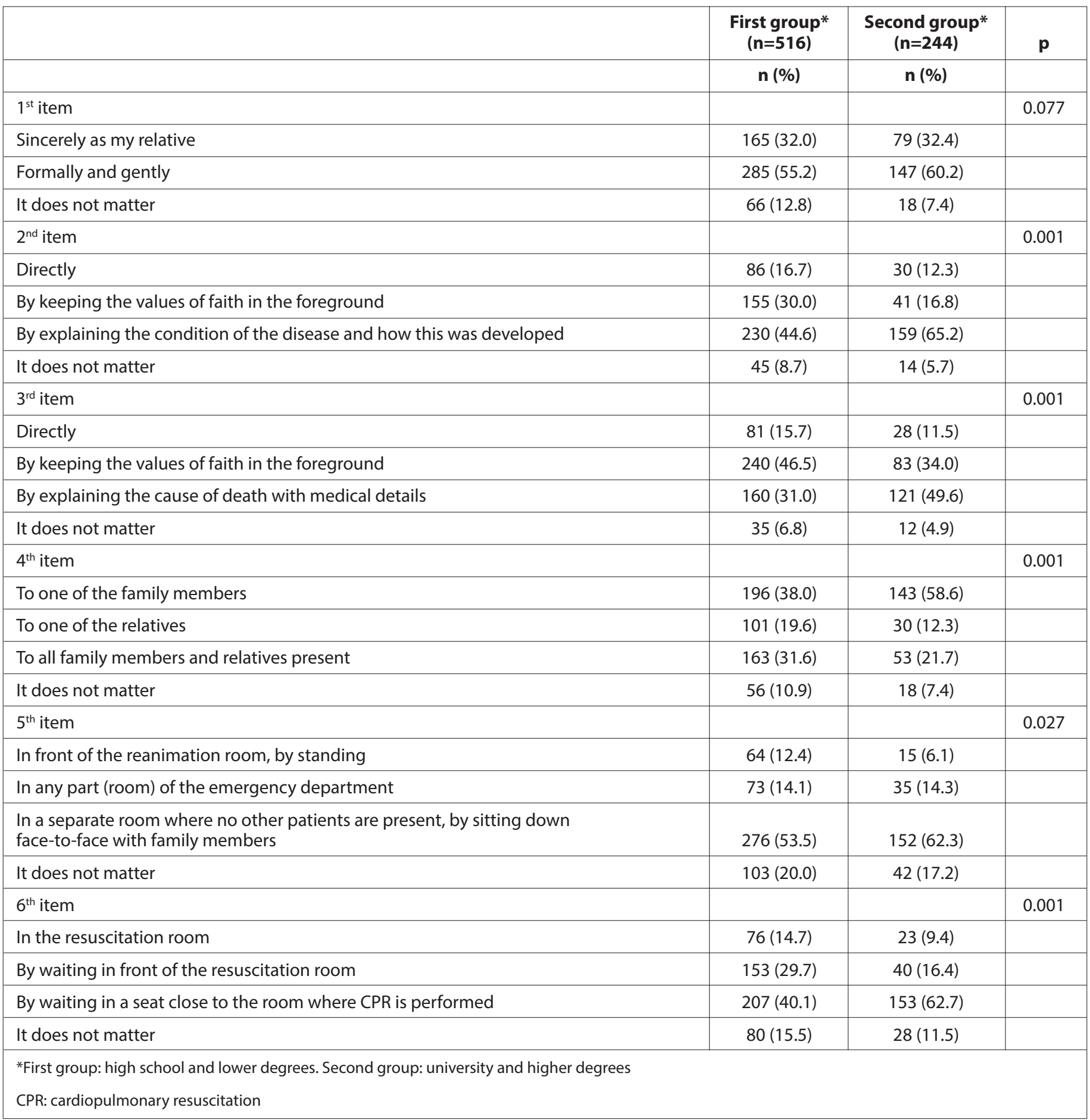

ic has been provided. One example of such technique is the SPIKES (Set the stage, Perception, Inform, Knowledge, Empathy, Summary and Strategy) protocol, which describes six steps of communication (13). The SPIKES protocol was developed in 2000 by an oncologist to train providers in delivering bad news (14). In 2005, the GRIEV_ING (Gather, Resources, Identify, Educate, Verify, Space, Inquire, Nuts and Bolts, Give) educational intervention was developed and tested by emergency physicians (15). Another study by Park et al. (4) described physicians' experience and designed an educational program for physician's confidence and competence in bad news and death notification.

Recent studies have focused on training doctors to improve their communication skills.

In the literature, a number of methods have been described how to break the bad news to a patient or his/her relatives. One of these is the $A B C D E$ model, which consists of five steps based on the initials 
of advanced preparation, build a therapeutic relationship and environment, communicate well, deal with patient and family reactions, and encourage and validate emotions (16). Another method is the SPIKES protocol that was developed by an oncologist in 2000 (14). BREAKS (Background, Rapport, Exploring, Announce, Kindling, Summarize), which was developed by oncologists and anesthesiologists in 2010, is another method used for BBN (17). Based on all three models, they were formed and developed by either internal medicine or oncologists and anesthesiologists. However, emergency physicians, in contrast to oncologists and anesthesiologists, often encounter the patient's family for the first time or do not have enough background information about the family $(4,18)$. In addition, the emergency physician does not have enough time for both preparing the family and preparing himself/herself for BBN (4).

GRIEV_ING is a protocol that is developed by emergency physicians (15). Both GRIEV_ING and other protocols have been developed to establish good communication strategies with patients and their family members in case of BBN. In our study, we asked ED patients as emergency physicians about what might be the facilitating factors for BBN in addition to how good communication should be. Participants in our study reported that they preferred good communication to be formal and gentle. GRIEV_ING and other protocols did not take into account whether the educational status of the individual or the family influenced the preferred language of communication. Participants in our study were divided into two groups according to their educational status, and we found that the language of communication they preferred varied according to educational status. Most of the participants in the second group (university or higher degrees) indicated that they would like to have BBN with medical details. In the G (Gather) of GRIEV_ING, it is recommended that the physician should gather the whole family together to inform them at once. In our study, in response to the item "To whom should a doctor tell the death of the patient to?", participants most frequently answered as 'to one of the family members' and less frequently as "to all family members and relatives present". Even participants in the second group (university and higher degrees) according to educational status answered as 'to one of the family members' more frequently.

In the S (Setting up the interview) of SPIKES, it is indicated that BBN should be conducted by sitting down in a room. Similar to SPIKES, participants answered the item 'Where should a doctor tell the death of the patient?' as "by sitting down face to face with family members, in a separate room where no other patients are present".

A similar study to our work, but with fewer participants and single center, conducted by Aminiahidasti et al. (3) in 2016 found that $83.7 \%$ of the participants said that a hospital corridor is not appropriate for BBN, and $52.30 \%$ of the participants pointed out that bad news should be delivered in a private room. These results are compatible with our study results. In a study investigating the effect of the patient's educational level on BBN conducted by Rao et al. (19), participants divided into two groups as high and low educational status and participants with high educational status preferred that BBN must be disclosed, and this difference is statistically significant. In our study, similar to the results by Rao et al. (19), participants with high educational status preferred to know all bad news in medical details. Similar results were concluded by Parker et al. (20) with 351 patients with cancer, and participants with high educational status wanted to know the bad news in more medical details.

Another issue that we questioned in our study was watching CPR or whether the family members prefer to be present during CPR. Although there are studies with different conclusions about this topic in the literature, it is thought that the mourning process may be more easily overcome by the presence of family members during CPR (2123). In contrast, in our study, participants reported that they preferred not to stay in the room where CPR was performed, but rather wait in a seat close to the room where CPR was performed. This result was the same for both groups.

\section{Study limitations}

Our study is limited by the number of patients.

\section{Conclusion}

Breaking bad news is a difficult task that needs to be overcome with less damage, for both patients and emergency physicians who deliver the news. Emergency physicians who often encounter this condition should learn good communication strategies and should consider the educational status of patients. Ideally, bad news should be delivered to one of the family members by sitting down face-toface in a room. It should also be taken into account that the patients may want to know the medical reasons of the condition they are in. Another finding of our study is that during CPR to one of the family members of the participants, they often prefer to wait in a room close to the CPR room rather than watch the CPR procedure.

Ethics Committee Approval: Ethics committee approval was received for this study from the Ethics Committee of Bitlis Eren University. (2017/02-V.)

Informed Consent: Written informed consent was obtained from the participants.

Peer-review: Externally peer-reviewed.

Author Contributions: Concept - R.G., G.E., AA.; Design - E.K.K., H.O.; Supervision - M.IS., A.A.; Resources - R.G., G.E., A.A.; Materials - E.K.K., R.G.; Data Collection and/or Processing - H.O., A.G.P.; Analysis and/or Interpretation - G.E., E.K.K., R.G.; Literature Search - H.O.; Writing Manuscript - A.A.; Critical Review - A.G.P., E.K.K.

Conflict of Interest: The authors have no conflict of interest to declare.

Financial Disclosure: The authors declared that this study has received no financial support.

\section{References}

1. Arbabi M, Rozdar A, Taher M, Shirzad M, Arjmand M, Ansari S, et al. Patients' preference to hear cancer diagnosis. Iran J Psychiatry. 2014; 9 : 8-13.

2. Toutin-Dias G, Daglius-Dias R, Scalabrini-Neto A. Breaking bad news in the emergency department: a comparative analysis among residents, patients and family members' perceptions. Eur J Emerg. 2016; 25: 71-6. [CrossRef]

3. Aminiahidashti H, Mousavi SJ, Darzi MM. Patients' Attitude toward Breaking Bad News; a Brief Report. Emerg (Tehran). 2016; 4: 34-7. 
4. Park I, Gupta A, Mandani K, Haubner L, Peckler B. Breaking bad news education for emergency residents: A novel training module using simulation with the SPIKES protocol. J Emerg Trauma Shock. 2010; 3: 385-8. [CrossRef]

5. Saaiq M, Uz-Zaman K. Breaking Bad News In Emergency: How Do We Approach It? Ann Pak Inst Med Sci. 2006; 2: 72-4.

6. Atesci FC, Oguzhanoglu NK, Baltalarli B, Karadag F, Ozdel O, Karagoz N. Psychiatric disorders in Cancer Patients and Associated Factors. Turk Psikiyatri Derg. 2003; 14: 145-52.

7. Naik SB. Death in the hospital: Breaking the bad news to the bereaved family. Indian J Crit Care Med. 2013; 17: 178-81. [CrossRef]

8. Dosanjh S, Barnes J, Bhandari M. Barriers to breaking bad news among medical and surgical residents. Med Educ. 2001; 35: 197-205. [CrossRef]

9. Martis L, Westhues A. A synthesis of the Literature on Breaking Bad News or Truth Telling: Potential for Research in India. Indian J Palliat Care. 2013; 19: 2-11. [CrossRef]

10. Goncalves F, Marques Á, Rocha S, Leitão P, Mesquita T, Moutinho S. Breaking bad news: experiences and preferences of advanced cancer patients at a Portuguese oncology centre. Palliat Med. 2005; 19: 526-31. [CrossRef]

11. Tuckett AG. Truth-telling in clinical practice and the arguments for and against: A review of the literature. Nurs Ethics. 2004; 11:500-13. [CrossRef]

12. Abbaszadeh A, Ehsani SR, Bejgani J, Kaji MA, Dopolani FN, Nejati A, et al. Nurses' perspectives on breaking bad news to patients and their families: a qualitative content analysis. J Med Ethics Hist Med. 2014; 7: 18.

13. Ferreira da Silveira FJ, Botelho CC, Valadão CC. Breaking bad news: doctors' skills in communicating with patients. Sao Paulo Med J. 2017; 135: 323-31. [CrossRef]
14. Baile WF, Buckman R, Lenzi R, Glober G, Beale EA, Kudelka AP. SPIKES-A six-step protocol for delivering bad news: Application to the patient with cancer. Oncologist. 2000; 5: 302-11. [CrossRef]

15. Hobgood C, Harward D, Newton K, Davis W. The educational intervention 'GRIEV_ING' improves the death notifaction skills of residents. Acad Emerg Med. 2005; 12: 296-301. [CrossRef]

16. Rabow MW, McPhee SJ. Beyond breaking bad news: how to help patients who suffer. West J Med. 1999; 171: 260-3.

17. Narayanan V, Bista B, Koshy C. 'BREAKS' protocol for Breaking Bad News. Indian J Palliat Care. 2010; 16: 61-5. [CrossRef]

18. Williams AG, O'Brien DL, Laughton KJ, Jelinek GA. Improving services to bereaved relatives in the emergency department: Making healthcare more human. Med J Aust. 2000; 173: 480-3.

19. Rao A, Sunil B, Ekstrand M, Heylen E, Raju G,mShet A. Breaking Bad News: Patient Preferences and the Role of Family Members when Delivering a Cancer Diagnosis. Asian Pac J Cancer Prev. 2016; 17: 1779-84. [CrossRef]

20. Parker PA, Baile W, Moor C, Lenzi R, Kudelka AP, Cohen L. Breaking bad news about cancer: Patients' preferences for communication. J Clin Oncol. 2001; 19: 2049-56. [CrossRef]

21. Tsai E. Should family members be present during cardiyopulmonary resuscitation? N Eng J Med. 2002; 346: 1019-21. [CrossRef]

22. McClenathan BM, Torrington KG, Uyehara CFT. Family member presence during cardiopulmonary resuscitation: a survey of US and International critical care professionals. Chest. 2002; 122: 2204-11. [CrossRef]

23. Jabre P, Vanessa B, Azoulay E, Jacob L, Bertrand L and Lapostolle F. Family Presence during Cardiyopulmonary Resuscitation. N Eng J Med. 2013; 368: 1008-18. [CrossRef] 